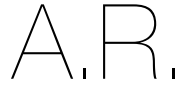

1 Escola Superior de Tecnologia da Saúde de Coimbra,

Rua 5 de Outubro,

3046-854 Coimbra,

Portugal

2 Unidade de Nutrição e Dietética do Hospital

Pediátrico do Centro

Hospitalar Universitário de

Coimbra,

Praceta Prof. Mota Pinto,

3000-075 Coimbra,

Portugal

*Endereço para correspondência

Ema Monteiro

Rua 5 de Outubro,

3046-854 Coimbra, Portuga

Histórico do artigo:

Recebido a 23 de agosto de 2019 Aceite a 26 de outubro de 2019

\title{
DEFICIÊNCIA DE VITAMINA D EM CRIANÇAS COM DOENÇA CELÍACA
}

\author{
VITAMIN D DEFICIENCY IN CHILDREN WITH CELIAC DISEASE
}

Ema Monteiro'*; Nanci Baptista2; Ana Faria'; Helena Loureiro'

RESUMO

INTRODUÇÃO: A doença celíaca é cada vez mais prevalente em todo o mundo. No entanto, até ao momento, o único tratamento é a prática de uma dieta isenta de glúten. O acompanhamento dos doentes celíacos ao longo da vida é fundamental uma vez que podem surgir diversas complicações de saúde. Uma das consequências estudadas é a deficiência em vitamina D.

OBJETIVOS: Analisar a existência de deficiência em vitamina D em crianças diagnosticadas com doença celíaca.

METODOLOGIA: Analisar a literatura publicada na base de dados eletrónica Pubmed nos últimos 10 anos utilizando os termos de pesquisa "vitamin D deficiency" e "celiac disease". A pesquisa foi realizada entre dezembro 2018 e fevereiro 2019.

CONCLUSÕES: Até ao momento os estudos ainda não apresentam resultados conclusivos relativamente à existência de deficiência em vitamina $\mathrm{D}$ em crianças com doença celíaca bem como à necessidade de dosear periodicamente esta vitamina ao longo da infância.

\section{PALAVRAS-CHAVE}

Crianças, Deficiência em vitamina D, Doença celíaca

\section{ABSTRACT}

INTRODUCTION: Celiac disease is becoming more prevalent worldwide. However, the only treatment available so far is the practice of a gluten free diet. A regular follow up on the celiac patients is essential as they are more prone to develop health complications. Vitamin D deficiency is one of the consequences pointed out by studies.

OBJECTIVES: Analyse the existence of Vitamin D deficiency in children diagnosed with celiac disease.

METHODOLOGY: Analyse the published literature in the electronic database Pubmed in the last 10 years using the search terms "vitamin D deficiency" and "celiac disease". The research started in December 2018 up until February 2019.

CONCLUSIONS: So far, the studies haven't shown any correlation between children with celiac disease and vitamin D deficiency, nor as to the need of suplementation with vitamin D throughout childhood.

KEYWORDS

Children, Vitamin D deficiency, Celiac disease

\section{INTRODUÇÃo}

A primeira descrição da doença celíaca (DC) surgiu no século I e II D.C. pelo médico Aretaeus. No entanto, foi em 1887 que Samuel Gee fez a primeira descrição de sintomatologia como alterações das fezes, fraqueza muscular, distensão abdominal e atraso de crescimento. Adicionalmente, Gee também salientou que a cura talvez estivesse relacionada com a dieta (1-5).

Mais tarde, em 1908, Herter percebeu que as gorduras provenientes da alimentação eram melhor toleradas do que os hidratos de carbono e, em 1918, concluiu que havia uma baixa tolerância ao pão. Em 1921, Howland identificou a intolerância aos hidratos de carbono e Haas propôs uma dieta restrita em hidratos de carbono com exceção do consumo de bananas (1).

Porém foi apenas durante a segunda guerra mundial, que o pediatra Wim Dicke mostrou que crianças celíacas que tinham sido alvo de restrições alimentares tinham melhorado significativamente os sintomas (1, 4-6).

A DC ou sprue celíaco ou enteropatia sensível ao glúten ou sprue não tropical é uma doença autoimune crónica caracterizada por uma atrofia das vilosidades e por alterações inflamatórias da mucosa do intestino delgado após a ingestão de glúten em indivíduos geneticamente suscetíveis. O único tratamento existente é a evicção total do glúten durante toda a vida $(7,8)$.

O glúten é um grupo de proteínas presentes nos cereais, tais como trigo (gliadina), centeio (secalina) e cevada (orceína) $(7,9,10)$. Apesar da aveia não conter glúten, durante o processamento dos cereais pode haver risco de contaminação com o trigo. Por outro lado, existe ainda um pequeno grupo de doentes celíacos (<5\%) que também 
é intolerante a este cereal. Assim, recomenda-se que um doente celíaco não ingira aveia nos primeiros meses de tratamento e, posteriormente, inclua apenas aveia pura, ou seja, onde a ausência de glúten está garantida (8).

O teor de glúten nos alimentos é regulado pelo Codex Alimentarius, e este define que os alimentos isentos de glúten têm de apresentar valores inferiores ou iguais a 20 miligramas por quilograma de produto (7).

A prevalência mundial da DC é cerca de $1 \%$, variando a proporção de indivíduos diagnosticados entre os vários países (11). Recentemente um estudo avaliou a prevalência em quatro países europeus (Finlândia, Itália, Irlanda do Norte e Alemanha) e prevalência média foi também de $1 \%$ (12).

Em Portugal, o único estudo realizado foi publicado em 2006, e referiu que existia uma prevalência de 1:134 indivíduos (13).

Nos últimos anos os estudos têm demonstrado um aumento do diagnóstico de novos casos. Se por um lado, isto pode ser explicado pela introdução de técnicas endoscópicas gastrointestinais para a elaboração de biopsias (década de 1970), por outro lado também foram identificadas duas moléculas de antigénio leucocitário humano (HLA) associadas à DC - HLA-DQ2 (final da década de 1980) e HLA-DQ8 (início da década de 1990) - que permitiram criar análises sanguíneas altamente sensíveis e específicas $(2,8,14,15)$.

Apesar destes argumentos, as evidências sugerem a existência de um aumento real da prevalência da DC causada por fatores ambientais, como por exemplo a quantidade de glúten na dieta da criança, infeções virais e a latitude da habitação $(2,15,16)$.

Atualmente sabe-se que a DC pode manifestar-se em qualquer idade $(8,17)$. De acordo com a quantidade de glúten ingerida e a resposta imunitária do indivíduo, a sintomatologia pode aparecer em semanas ou meses (3).

Na infância é mais frequente encontrar a apresentação clássica da DC, caracterizada principalmente por sintomas gastrointestinais (Tabela1) que se iniciam entre os 6 e os 24 meses, após a introdução do glúten na dieta. As manifestações mais comuns na criança são o atraso no desenvolvimento e crescimento, perda de peso e de massa muscular, anemia por deficiência em ferro, diarreia crónica, vómitos, distensão abdominal, edema e irritabilidade $(7,8)$. No entanto tem surgido uma tendência de início tardio da DC, envolvendo crianças mais velhas (5 a 7 anos), adolescentes e adultos com uma apresentação não clássica, isto é, com sintomas gastrointestinais sem sinais de má absorção e/ou manifestações extraintestinais $(3,10)$.

As deficiências de vitaminas e oligoelementos podem desenvolver-se devido à baixa ingestão, alterações no metabolismo e/ou alterações na absorção por perda da superfície de absorção e aumento da permeabilidade intestinal. Uma das carências investigadas é a vitamina D (15, 18-20).

Existem duas fontes de vitamina D: dieta e síntese cutânea. A principal fonte de vitamina $D$ é produzida na pele após a exposição direta à radiação ultravioleta do sol. É por este motivo que é chamada a vitamina do sol bem como é considerada uma hormona. No entanto esta vitamina pode também ser encontrada em vários alimentos: os alimentos vegetais contêm vitamina D2 (ergocalciferol) e os alimentos de origem animal, como peixe gordo (salmão, cavala e sardinha), fígado, gema de ovo e óleo de fígado de bacalhau, contêm vitamina D3 (colecalciferol) $(19,21)$. Após a sua obtenção na alimentação e produção na pele, as vitaminas D2 e D3 são hidroxiladas no fígado em 25-hidroxivitamina D (principal forma de vitamina $D$ em circulação e armazenamento) e seguidamente no rim em 1,25-dihidroxivitamina $D$ (forma biologicamente ativa). Posteriormente a vitamina $\mathrm{D}$, sendo lipofilica, pode ser armazenada no tecido adiposo $(19,21,22)$.

\section{Tabela 1}

Sintomas gastrointestinais e extraintestinais da doença celíaca na infância

\begin{tabular}{ll} 
SINTOMAS GASTROINTESTINAIS & \multicolumn{1}{c}{ SINTOMAS EXTRAINTESTINAIS } \\
Anorexia & Anemia por deficiência de ferro \\
\hline Atraso de crescimento & $\begin{array}{l}\text { Deficiência de ácido fólico e / ou vitamina } \\
\text { B12 }\end{array}$ \\
\hline Perda de peso & Ansiedade \\
\hline Diarreia & Depressão \\
\hline Obstipação crónica & Ataxia Cerebelar \\
\hline Distensão abdominal & Dores de cabeça recorrentes \\
\hline Dor abdominal crónica & Convulsões \\
\hline Vómitos & Fadiga crónica \\
\hline & Puberdade tardia \\
\hline & Baixa estatura \\
\hline Fraturas ósseas \\
\hline Osteopenia ou osteoporose \\
\hline Artralgia e Artrite \\
\hline Neuropatia periférica \\
\hline Dermatite Herpetiforme \\
\hline Estomatite aftosa \\
\hline Alterações do esmalte dentário \\
Níveis moderadamente elevados de \\
alanina aminotransferase e aspartato \\
aminotransferase
\end{tabular}

Adaptado/traduzido com autorização de Springer Nature Customer Service Centre GmbH: Springer Nature Reviews Gastroenterology \& Hepatology, Coeliac disease and gluten-related disorders in childhood, Vriezinga, S.L., Schweizer, J.J., Koning, F., \& Mearin, M.L. - Copyright 2015 (licença 4647111308476).

O doseamento de vitamina $\mathrm{D}$ deve ser determinado pelo valor plasmático de 25-hidroxivitamina $\mathrm{D}$ uma vez que o seu tempo de semi-vida é de 2 a 3 semanas enquanto a 1,25-dihidroxivitamina D de apenas 4 horas. Adicionalmente a forma ativa de vitamina $D$ é influenciada pelos níveis de cálcio e fósforo que estimulam a secreção da paratormona e que consequentemente aumentam a produção renal de 1,25-dihidroxivitamina $D$. As recomendações mais recentes para crianças indicam que os níveis plasmáticos de vitamina $D$ devem ser superiores a $10-12 \mathrm{ng} / \mathrm{ml}(25-30 \mathrm{nmol} / \mathrm{L})(23)$.

A deficiência de vitamina $D$ pode dever-se à geografia (latitude, estação do ano, sombra do dia e poluição do ar), menor exposição ao sol, uso de roupa e protetor solar, envelhecimento, pele escura, baixa ingestão alimentar e menor absorção (10, 15, 20). Independentemente do motivo, em caso de deficiência prolongada podem surgir alterações na mineralização óssea, levando ao aparecimento de raquitismo e osteopenia em crianças $(10,24)$.

Com a descoberta dos recetores da vitamina $\mathrm{D}$ em diversos tecidos, tem-se estudado a sua relação com várias doenças, como diabetes, doenças cardiovasculares, cancro (linfoma, cancro colo-rectal, mama, próstata, ovários), doenças psiquiátricas (esquizofrenia, depressão e demência), doenças infeciosas, alergias, asma, processos autoimunes (esclerose múltipla, lúpus eritematoso sistêmico, artrite reumatoide) e inflamatórios (10, 18, 19, 25-27).

Uma vez que as fontes alimentares não fortificadas em vitamina $D$ não são consumidas habitualmente pelas crianças, esta deficiência torna-se particularmente importante na população infantil devido ao seu rápido desenvolvimento e crescimento $(10,22)$.

Atualmente ainda não existe consenso no que diz respeito ao doseamento de vitaminas lipossolúveis em doentes celíacos nem à toma de suplementos vitamínicos ou à avaliação frequente de vitaminas lipossolúveis em crianças celíacas $(15,20)$. 


\section{METODOLOGIA}

A pesquisa foi realizada na base de dados eletrónica PubMed entre dezembro 2018 e fevereiro 2019. A pesquisa abrangeu artigos publicados entre 2009 e 2019 e os termos de pesquisa utilizados foram ""vitamin D deficiency" AND "celiac disease"".

Foram incluídos artigos publicados na língua inglesa e espanhola entre 2009 e 2019 realizados em seres humanos entre os 0 aos 18 anos. Os estudos que não analisaram os níveis plasmáticos de vitamina D foram excluídos.

A fundamentação teórica do artigo não seguiu o método de busca supracitado.

\section{RESULTADOS}

Na primeira pesquisa foram recuperados 144 artigos. Após a aplicação dos vários filtros (idade, língua, anos de publicação e população humana) obtiveram-se 32 artigos. Seguidamente analisou-se o título, o resumo e o texto completo e foram selecionados 12 estudos.

O objetivo deste estudo foi analisar a presença de deficiência em vitamina D em crianças com DC.

Considerando os 12 artigos selecionados, a população total estudada foi de 979 crianças. Destes estudos, 1 foi conduzido no Chile, 1 na Arábia Saudita, 1 no Canadá, 1 na Holanda, 1 na Eslovénia, 2 nos EUA, 2 na Turquia e 1 estudo utilizou população de Israel e Espanha. A duração dos estudos variou de 3 meses a 17 anos.

\section{ANÁLISE CRÍTICA}

Dos 12 estudos analisados, apenas 3 apresentaram grupo controlo. O estudo de Villanueva, Maranda e Nwosu publicado em 2012 (28) avaliou um grupo de 24 crianças celíacas recém-diagnosticadas (62,5\% do sexo feminino) e um grupo de 50 crianças saudáveis (42\% do sexo feminino) e não encontrou diferenças significativas entre os dois grupos no que diz respeito aos níveis de vitamina $\mathrm{D}$ (o primeiro grupo apresentava $27,58 \pm 9,91 \mathrm{ng} / \mathrm{ml}$ enquanto que o grupo controlo $26,20 \pm 10,4 \mathrm{ng} /$ $\mathrm{ml})$. Neste estudo os pontos de corte utilizados consideraram que ambos estavam com níveis suficientes de vitamina D. Adicionalmente verificaram também que não existiam diferença significativas dos níveis médios de vitamina $D$ entre as crianças saudáveis e crianças celíacas quando ajustadas para o Índice de Massa Corporal (IMC), mas que em ambos os grupos as crianças obesas apresentavam níveis mais baixos de vitamina $D$ em comparação com crianças não obesas. O motivo poderá estar relacionado com o facto da vitamina $D$ ser lipossolúvel e consequentemente ficar acumulada no tecido adiposo. Assim, o IMC elevado deverá ser um parâmetro a considerar em estudos futuros uma vez que poderá aumentar o risco de deficiência desta vitamina.

Lerner et al. (25) avaliaram 110 celíacos provenientes de Espanha e Israel e 56 crianças com sintomatologia limitada a dor abdominal de dor abdominal provenientes de Israel. A hipovitaminose verificou-se em todos os grupos, sendo que as crianças celíacas de Israel apresentaram $33,3 \%$ de deficiência (<20 ng/mL), de Espanha 16,95\% e o grupo controlo 29,63\%. O maior nível de deficiência nas crianças israelitas pode ser explicada pela pigmentação da pele e hábitos culturais que influenciam o vestuário. Assim, será importante realizar novos estudos que incluam no grupo controlo ambas as populações.

Mais recentemente, em 2018, o estudo de Tokgöz, Terlemez e Karul (20) avaliou um grupo de 52 crianças recém-diagnosticadas com DC (51,9\% do sexo feminino) e um grupo de 50 crianças saudáveis (50\% do sexo feminino). Os níveis de vitaminas lipossolúveis foram avaliados em ambos os grupos e a única deficiência encontrada no grupo controlo foi vitamina D. Apesar disso, as crianças celíacas apresentaram níveis significativamente menores (92,3\% apresentou níveis insuficientes (<30ng/ml) e 61,5\% deficientes (<20ng/ml)) em comparação com o grupo controlo saudável (18\% apresentou níveis insuficientes e 4\% deficientes).

Em 2018 também foi publicado o estudo de Granfeldt et al. (10) que teve em conta o parâmetro IMC, e dos 16 celíacos $(81,25 \%$ sexo feminino) considerou 11 eutróficos, 4 com sobrepeso e 1 obeso. Neste estudo determinaram que 25\% apresentavam níveis suficientes de vitamina D (30-100 ng/ml), 50\% insuficiência (20$29 \mathrm{ng} / \mathrm{ml}$ ) e $25 \%$ deficiência (<20 ng/ml). No entanto, não foram encontradas diferenças significativas na concentração de vitamina $\mathrm{D}$ entre as diferentes classificações de IMC. Este resultado deverá ser analisando em estudos futuros devido à reduzida amostra.

O estudo de Topal et al. publicado em 2015 (29) acompanhou apenas um grupo de 52 crianças celíacas (63,5\% sexo feminino) e verificou que $51,9 \%$ apresentava deficiência $(<20 \mathrm{ng} / \mathrm{ml})$.

O estudo realizado por Fouda (30) analisou entre 2003 e 2012 crianças e adultos celíacos. Relativamente às 90 crianças com DC $(65,6 \%$ sexo

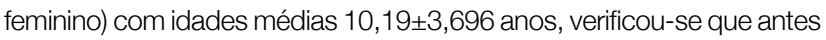
de introduzir uma dieta isenta de glúten 76,3\% apresentava deficiência ( $<50 \mathrm{nmol} / \mathrm{L})$ e $21,1 \%$ insuficiência (50 - <75 nmol/L) de vitamina D. Estes dados foram ainda mais elevados no adulto. Relativamente à densidade mineral óssea, esta encontrou-se baixa tanto nas crianças como nos adultos.

Por outro lado, o estudo de Imam, Ghazzawi, Murray e Absah (15) analisou os registos de 83 crianças $(61,4 \%$ sexo feminino) diagnosticadas entre 1995 e 2012 que apresentavam avaliação bioquímica de vitaminas lipossolúveis e verificou que nenhuma tinha deficiência grave em vitamina D (isto é $<10 \mathrm{ng} / \mathrm{ml}$ ) e apenas 11\% apresentava deficiência leve a moderada (10-19 ng/ml). Os autores concluíram que ou esta deficiência é incomum nesta população ou que o diagnóstico precoce pode ter permitido introduzir a dieta isenta de glúten cedo e evitar complicações de má absorção. Outra possibilidade pode estar relacionada ainda com o uso frequente de suplementos vitamínicos ou fortificação de alimentos. O estudo de Deora, Aylward, Sokoro e El-Matary publicado em 2017 (31) bem como o estudo de Wessels et al. de 2016 (32) dosearam a vitamina $\mathrm{D}$ em vários momentos. Ambos constataram maior número de crianças com deficiência em vitamina D no momento de diagnóstico. No entanto, a deficiência mantém-se em alguns casos, e segundo o estudo de Deora et al. (31) após 18 meses da introdução da dieta isenta de glúten, 57,4\% ainda apresentava deficiência. Isto pode ser explicado porque os níveis de vitamina $D$ são influenciados por outros fatores além da menor absorção, como época do ano e cor da pele. Os autores sugerem ser necessário fazer estudos controlados de maior dimensão e duração para se determinar o impacto dos vários parâmetros no risco de desenvolver deficiência em vitamina $D$ ao longo da vida $(31,32)$. Outro fator que foi alvo de investigação foi a adesão à dieta isenta de glúten. O estudo de Blazina, Bratanic, Campa, Blagus e Orel (33) comparou os dados de vitamina $\mathrm{D}$ com a adesão à dieta isenta de glúten. O grupo com dieta estrita há 2 anos continha 55 crianças $(72,7 \%$ sexo feminino) e o grupo sem dieta estrita era composto por 19 crianças (68,4\% do sexo feminino). Ambos os grupos apresentaram deficiência em vitamina $\mathrm{D}$, mas sem diferença significativa.

O estudo não forneceu dados que permitissem refletir acerca da prevalência de deficiência de vitamina D na população portuguesa pediátrica celíaca. Tal pode ser explicado pelo facto de atualmente ainda não ser protocolado o seu doseamento. Caso nos baseemos nos resultados do estudo que incluiu Espanha (25) devido à sua proximidade geográfica, podemos supor que existirá deficiência em Portugal. No entanto, seria importante realizar um estudo prospetivo controlado que tivesse em conta os níveis plasmáticos de vitamina $D$ 
bem como parâmetros como a idade, o género, o IMC, a estação do ano, a localização geográfica, o tempo de exposição solar, o uso de protetor solar e a adesão à dieta isenta de glúten.

A seleção dos artigos limitada às línguas inglesa e espanhola e a ausência de grupo controlo em alguns dos artigos encontrados são factos considerados como limitações a esta revisão.

\section{CONCLUSÕES}

A presente revisão evidencia que os vários estudos analisados mostram resultados díspares relativamente à presença de deficiência em vitamina D em doentes celíacos bem como à necessidade de avaliar os níveis plasmáticos desta vitamina ao longo da infância.

Até ao momento, há estudos que destacam que o diagnóstico precoce desta doença permite a introdução rápida de uma dieta isenta de glúten e consequentemente reduzir o risco de complicações de má absorção intestinal. No entanto, poucos estudos analisam a adesão à dieta a longo prazo e o impacto da mesma nos níveis de vitamina $D$.

Por outro lado existem estudos que destacam a necessidade de avaliar outros fatores, como a localização geográfica e estação do ano. A vitamina $D$ é maioritariamente obtida pela exposição direta da pele à radiação ultravioleta do sol. Caso não exista uma avaliação continua em vários momentos do ano os resultados poderão não ser precisos. Adicionalmente a cor da pele e a utilização de protetor solar também são parâmetros que deverão ser analisados uma vez que podem influenciar a capacidade de síntese cutânea.

Dos estudos analisados ainda houve destaque para a obesidade, que pode contribuir para a deficiência de vitamina $D$ uma vez que esta é lipossolúvel e fica armazenada no tecido adiposo.

Em suma, o número reduzido de estudos em idade pediátrica, o tamanho das amostras, a ausência de grupos de controlo bem como a dificuldade de analisar os vários fatores que influenciam os níveis de vitamina D (localização geográfica, cor da pele, obesidade, exposição solar, uso de protetor solar, ingestão alimentar de vitamina D e adesão à dieta isenta de glúten) conduzem à necessidade de realizar mais investigação de forma a encontrar estratégias para evitar a deficiência desta vitamina que tem apresentado cada vez mais funções essenciais à saúde.

\section{REFERÊNCIAS BIBLIOGRÁFICAS}

1. Losowsky MS. A history of coeliac disease. Dig Dis. 2008;26(2):112-20.

2. Tack GJ, Verbeek WH, Schreurs MW, Mulder CJ. The spectrum of celiac disease: epidemiology, clinical aspects and treatment. Nat Rev Gastroenterol Hepatol. 2010;7(4):204-13.

3. Fasano A, Catassi C. Coeliac disease in children. Best Pract Res Clin Gastroenterol. 2005;19(3):467-78.

4. Farrell RJ, Kelly CP. Celiac sprue. N Engl J Med. 2002;346(3):180-8.

5. Tanpowpong P, Camargo CA. Early-life vitamin D deficiency and childhood-onset coeliac disease. Public Health Nutr. 2014;17(4):823-6.

6. Anderson CM, French JM, Sammons HG, Frazer AC, Gerrard JW, Smellie JM. Coeliac disease; gastrointestinal studies and the effect of dietary wheat flour. Lancet. 1952;1(6713):836-42.

7. Ludvigsson JF, Leffler DA, Bai JC, Biagi F, Fasano A, Green PH, et al. The Oslo definitions for coeliac disease and related terms. Gut. 2013;62(1):43-52.

8. Bai JC, Ciacci C. World Gastroenterology Organisation Global Guidelines: Celiac Disease February 2017. J Clin Gastroenterol. 2017;51(9):755-68.

9. Vriezinga SL, Schweizer JJ, Koning F, Mearin ML. Coeliac disease and gluten-related disorders in childhood. Nat Rev Gastroenterol Hepatol. 2015;12(9):527-36.

10. Granfeldt M G, Zapata F D, Muñoz R S, Bello M MF, Victoriano R M, Mennickent $C$ S, et al. Vitamin D concentrations in children and adolescents with celiac disease. Rev Chil Pediatr2018. p. 499-505.
11. Fasano A, Berti I, Gerarduzzi T, Not T, Colletti RB, Drago S, et al. Prevalence of celiac disease in at-risk and not-at-risk groups in the United States: a large multicenter study. Arch Intern Med. 2003;163(3):286-92.

12. Mustalahti K, Catassi C, Reunanen A, Fabiani E, Heier M, McMillan S, et al. The prevalence of celiac disease in Europe: results of a centralized, international mass screening project. Ann Med. 2010;42(8):587-95.

13. Antunes H, Abreu I, Nogueiras A, Sá C, Gonçalves C, Cleto P, et al. [First determination of the prevalence of celiac disease in a Portuguese population]. Acta Med Port. 2006;19(2):115-20.

14. Sollid LM, Markussen G, Ek J, Gjerde H, Vartdal F, Thorsby E. Evidence for a primary association of celiac disease to a particular HLA-DQ alpha/beta heterodimer. J Exp Med. 1989;169(1):345-50.

15. Imam MH, Ghazzawi Y, Murray JA, Absah I. Is it necessary to assess for fat-soluble vitamin deficiencies in pediatric patients with newly diagnosed celiac disease? J Pediatr Gastroenterol Nutr. 2014;59(2):225-8.

16. Lohi S, Mustalahti K, Kaukinen K, Laurila K, Collin P, Rissanen H, et al. Increasing prevalence of coeliac disease over time. Aliment Pharmacol Ther. 2007;26(9):1217-25. 17. Thomas PC, Tighe MP, Beattie RM. Coeliac disease in children. BMJ. 2018;363:k3932.

18. O'Malley T, Heuberger R. Vitamin D status and supplementation in pediatric gastrointestinal disease. J Spec Pediatr Nurs. 2011;16(2):140-50.

19. Ahlawat R, Weinstein T, Pettei MJ. Vitamin $D$ in pediatric gastrointestinal disease. Curr Opin Pediatr. 2017;29(1):122-7.

20. Tokgöz Y, Terlemez S, Karul A. Fat soluble vitamin levels in children with newly diagnosed celiac disease, a case control study. BMC Pediatr. 2018;18(1):130.

21. Wintermeyer E, Inle C, Ehnert S, Stöckle U, Ochs G, de Zwart P, et al. Crucial Role of Vitamin D in the Musculoskeletal System. Nutrients. 2016;8(6).

22. Misra M, Pacaud D, Petryk A, Collett-Solberg PF, Kappy M, Society DaTCotLWPE. Vitamin D deficiency in children and its management: review of current knowledge and recommendations. Pediatrics. 2008;122(2):398-417.

23. Bouillon R, Carmeliet G. Vitamin D insufficiency: Definition, diagnosis and management. Best Pract Res Clin Endocrinol Metab. 2018;32(5):669-84.

24. Nwosu BU, Maranda L. Vitamin D Status and Adiposity in Pediatric Malabsorption Syndromes. Digestion. 2015;92(1):1-7.

25. Lerner A, Shapira Y, Agmon-Levin N, Pacht A, Ben-Ami Shor D, López HM, et al. The clinical significance of $25 \mathrm{OH}$-Vitamin D status in celiac disease. Clin Rev Allergy Immunol. 2012;42(3):322-30.

26. Ramagopalan SV, Goldacre R, Disanto G, Giovannoni G, Goldacre MJ. Hospital admissions for vitamin $D$ related conditions and subsequent immune-mediated disease: record-linkage studies. BMC Med. 2013;11:171.

27. Yin K, Agrawal DK. Vitamin D and inflammatory diseases. J Inflamm Res. 2014;7:69-87. 28. Villanueva J, Maranda L, Nwosu BU. Is vitamin D deficiency a feature of pediatric celiac disease? J Pediatr Endocrinol Metab. 2012;25(5-6):607-10.

29. Topal E, Çatal F, Yildirim Acar N, Ermistekin H, Sinanoglu MS, Karabiber H, et al. Vitamin and mineral deficiency in children newly diagnosed with celiac disease. Turk J Med Sci. 2015;45(4):833-6.

30. Fouda MA. Celiac disease-related osteopathy among Saudi celiac patients: Are we adherent to recommendations? Saudi J Gastroenterol. 2017;23(5):291-5.

31. Deora V, Aylward N, Sokoro A, El-Matary W. Serum Vitamins and Minerals at Diagnosis and Follow-up in Children With Celiac Disease. J Pediatr Gastroenterol Nutr. 2017;65(2):185-9.

32. Wessels MM, van Veen II, Vriezinga SL, Putter H, Rings EH, Mearin ML. Complementary Serologic Investigations in Children with Celiac Disease Is Unnecessary during Follow-Up. J Pediatr. 2016;169:55-60.

33. Blazina S, Bratanic N, Campa AS, Blagus R, Orel R. Bone mineral density and importance of strict gluten-free diet in children and adolescents with celiac disease. Bone. 2010;47(3):598-603. 